\title{
Rescuer fatigue does not correlate to energy expenditure during simulated basic life support
}

\author{
ROMAN SKULEC ${ }^{1,2,3}$, ANATOLIJ TRUHLAR ${ }^{1,4}$, VLADIMIR VONDRUSKA ${ }^{5}$, RENATA PARIZKOVA $^{1}$, JAROSLAVA \\ DUDAKOVA ${ }^{3}$, DAVID ASTAPENKO ${ }^{1}$, TOMAS SUCHY ${ }^{1}$, CHRISTIAN LEHMANN ${ }^{6}$, VLADIMIR CERNY $Y^{1,2,7}$ \\ ${ }^{1}$ Department of Anesthesiology and Intensive Care, Charles University in Prague, Faculty of Medicine in \\ Hradec Kralove, University Hospital Hradec Kralove, Hradec Kralove, Czech Republic \\ ${ }^{2}$ Department of Anesthesiology, Perioperative Medicine and Intensive Care, J.E. Purkinje University, Masaryk Hospital, Usti nad Labem, Czech Republic \\ ${ }^{3}$ Emergency Medical Service of the Central Bohemian Region, Beroun, Czech Republic \\ ${ }^{4}$ Hradec Kralove Region Emergency Medical Services, Hradec Kralove, Czech Republic \\ ${ }^{5} 1$ st Department of Internal Medicine, Department of Sports Medicine, Charles University in Prague, Faculty of \\ Medicine in Hradec Kralove, University Hospital Hradec Kralove, Hradec Kralove, Czech republic \\ ${ }^{6}$ Department of Anesthesia, Pain Management and Perioperative Medicine, Dalhousie University, Halifax, Nova Scotia, Canada \\ ${ }^{7}$ Department of Research and Development and Department of Anesthesiology and Intensive Care, Charles University in \\ Prague, Faculty of Medicine in Hradec Kralove, University Hospital Hradec Kralove, Hradec Kralove, Czech Republic
}

Corresponding author:

Roman Skulec

Department of Anesthesiology and Intensive Care

Charles University in Prague, ZZSSCK, Prof. Veseleho 461

Beroun 266 01, Czech Republic

Phone: 00420777577497

E-mail: skulec@email.cz

\section{ABSTRACT}

It is known that providing basic life support (BLS) may be limited by the physical capabilities of rescuers. The other factor that may affect BLS quality is its energy expenditure. Therefore, we decided to compare the energy expenditure of standard BLS with a compression-ventilation ratio of 30:2 (S-BLS) and compression-only BLS (CO-BLS) and assess the sensation of fatigue and perceived exertion associated with these activities.

Methods. We conducted a simulation study on 10 healthy volunteers using a resuscitation manikin. Participants were randomly assigned to start with CO-BLS or with SBLS, in accordance with recent guidelines. Later, every individual provided the other type of BLS. BLS was terminated in the event of exhaustion, impossibility to retain high-quality BLS or after 30 minutes of BLS. Energy expenditure was expressed as relative oxygen consumption (VO2/ $\mathrm{kg}$ ) and area under the curve of all $\mathrm{VO} 2 /$ $\mathrm{kg}$ measurements during each BLS procedure indexed to one minute (AUCVO2/kg min). All participants completed a survey to assess perceived intensity of exertion by Borg, and sensation of general fatigue by visual analogue scale.
Results. Maximal VO2/kg (23.16 \pm 3.94 vs. $20.17 \pm 2.14 \mathrm{ml} / \mathrm{kg} / \mathrm{min}, \quad \mathrm{p}=0.049)$ and $\mathrm{AUCVO} 2 / \mathrm{kg} \min (18.90 \pm 3.13$ vs. $15.91 \pm 2.07 \mathrm{ml} / \mathrm{min} 3$; $\mathrm{p}=0.021)$ during $\mathrm{S}$ BLS were significantly higher compared to CO-BLS. Conversely, a more intense rate of perceived exertion (16.6 \pm 2.0 vs. $13.8 \pm 1.2$, $\mathrm{p}=0.001$ ) and sensation of general fatigue $(86.5 \pm 10.8$ vs. $75.0 \pm 14.3, \mathrm{p}=0.058)$ were associated with CO-BLS. Neither sensation of general fatigue, nor perceived exertion correlated with energy expenditure.

Conclusions. Energy expenditure of S-BLS was higher than of CO-BLS in our study, while sensation of fatigue and perceived exertion reflected the opposite association.

Key words: basic life support, energy expenditure, general fatigue

\section{INTRODUCTION}

In the case of recognized out-of-hospital cardiac arrest (OHCA), initiation of early bystander cardiopulmonary resuscitation (CPR) is of major importance. It should be started immediately and ensure delivery of high-quality chest compressions until arrival of emergency medical ser- vices (EMS). While trained rescuers are supposed to perform standard basic life support (S-BLS) with a compression-ventilation ratio of $30: 2$, those persons who are not trained or are unwilling to give rescue breaths are encouraged to provide compression-only BLS (CO-BLS). CO-BLS is also the first choice for telephone-guided CPR. (1) Among other factors, the quality and duration of BLS may be limited by the physical capabilities of rescuers. In recent guidelines, this topic is described briefly stating that chest compression depth may decrease soon due to rescuer fatigue. Thus, if there is more than one rescuer present, another rescuer should take over delivering CPR every $2 \mathrm{~min}$ to prevent fatigue. (1) However, evaluation of BLS feasibility in relation to the physical capabilities of providers can be assessed not only by subjective indicators (sensation of general fatigue, perceived intensity of exertion) but also by objective measurement of energy expenditure, expressed as oxygen consumption during exercise. (2) We believe that detailed and multimodal investigation of BLS from this perspective may improve the quality of simulation studies, increase its comparability and perhaps can affect further optimization of BLS procedures, including protocols for emergency medical dispatchers. Therefore, we performed 
the study comparing energy requirements, sensation of general fatigue and perceived intensity of exertion during CO-BLS and S-BLS performed by healthy volunteers on a resuscitation manikin. We hypothesize, that CO-BLS is associated with higher energy expenditure, and that higher energy expenditure is associated with higher degree of sensation of general fatigue and perceived intensity of exertion.

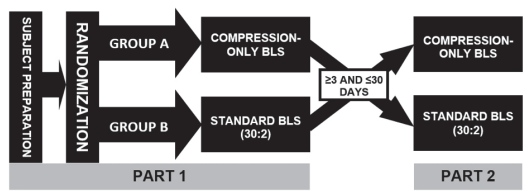

Figure 1. Flow-chart of the study protocol.

\section{MATERIALS AND METHODS}

We conducted an unblinded, randomized, cross-over study on healthy volunteers using a full body resuscitation manikin. The study was conducted in accordance with the Declaration of Helsinki and was approved by the local ethics committee of the University Hospital Hradec Kralove, Czech republic (reference number 201204 S18P, approved 2012-04-12). All participants signed informed consent before inclusion in the study. The study was conducted in the air conditioned, sports medicine laboratory of the 1st Department of Internal Medicine, Charles University in Prague, Faculty of Medicine in Hradec Kralove, University Hospital Hradec Kralove, Czech republic.

The volunteers were healthy medical students who passed a BLS course within the previous 6 months as part of their mandatory education. They stated that they were not involved in any regular sporting activities. All study procedures were performed in the morning. On the day, fasting $>2$ and $<6$ hours was required and none of the participants suffered from any intercurrent disease. All participants were informed about the purpose of the study and protocol details (figure 1). The volunteers were randomly assigned, using the envelope method, to start with CO-BLS (group A) or with S-BLS simulation (group B). In the second part of the study, which was realized $\geq 3$ and $\leq 30$ days after the first part, every individual performed the other type of BLS. BLS in both groups was delivered in accordance with the ERC guidelines from 2010. (1) Participants performed chest compressions while kneeling on the floor, right to the manikin's chest. Simulated CPR was performed on a Resusci Anne Advanced SkillTrainer (Laerdal Medical, Norway) manikin, which was connected to a PC with installed Laerdal Skill Reporting software (Laerdal Medical, Norway) providing real-time CPR performance feedback. It was set to maintain a compression rate of $100-120 / \mathrm{min}$ with the proportion of adequate compressions (depth of 5-6 cm followed by full decompression) $>80 \%$. The time taken to simulate two rescue breaths was set not to exceed $5 \mathrm{~s}$. As all volunteers were wearing a facemask connected to a spiroergometric machine for gas analysis during BLS, simulation of mouth-to-mouth ventilations had to be modified accordingly. During the pause for two ventilations, the students bent over just above the manikin's mouth but expired breath into the facemask. An investigator supervised course of every BLS session in order to maintain permanent high-quality CPR. All participants were continuously verbally motivated by supervisor every minute, even if the CPR quality was good. Moreover, every volunteer was immediately informed when the compression rate exceeded a predefined range and/or proportion of adequate compressions decreased $<80 \%$ for more than 30 seconds.

The criteria for BLS termination were as follows: feeling of severe exhaustion excluding further continuation of BLS, impossibility to retain high-quality $\mathrm{CPR}$ despite three consecutive warnings from the investigator, or high-quality CPR performed for at least 30 minutes.

Retrospectively, all participants completed a survey to assess perceived intensity of exertion by Borg, and sensation of general fatigue after protocol completion by visual analogue scale (0-100) in both groups. Participants were simply asked which BLS approach was less exhaustive and why. (3)

\section{Measurements}

Energy expenditure of BLS was expressed as oxygen consumption throughout the protocol. It was measured in all participants continuously using a spiroergometric monitoring system and gas analyser (Kardiospirox-W1, Junkalor, Hradec Kralove, Czech Republic). We also monitored heart rate (beats/min) and respiratory rate (breaths/min). For further analysis, we calculated relative oxygen consumption (VO2 $/ \mathrm{kg}, \mathrm{ml} / \mathrm{kg} / \mathrm{min}$ ) and area under the curve (AUC) of all VO2/kg measurements during each BLS procedure indexed to one minute (AUCVO2/kg $\mathrm{min}, \mathrm{ml} / \mathrm{min}$ ), to exclude the impact of different duration of BLS sessions between volunteers when evaluating AUC. One metabolic equivalent (MET) was set by convention to $3.5 \mathrm{ml}$ $\mathrm{O} 2 / \mathrm{kg} / \mathrm{min}$. (4) Respiratory exchange ratio was calculated as the ratio of the amount of $\mathrm{CO} 2$ produced to the amount of $\mathrm{O} 2$ consumed.

\section{Statistical analysis}

Sample size was calculated (with limited validity due to the pilot nature of the study) to detect a standardized difference of AUCVO2 $/ \mathrm{kg}$ min of 2.0 with $80 \%$ power using a cut off for statistical significance of 0.05 resulting in 10 participants in each group. All measurements, taken at 30-second intervals, were processed for statistical analysis. Mean values \pm standard deviation or percentages were calculated as necessary. Differences between groups were compared using the $\chi^{2}$ test. Statistical significance was calculated using the Fischer exact test for alternative variables, while significance for continuous variables was determined using the paired Student's t-test or Wilcoxon test and Kruskal-Wallis test, by the type of data distribution. The area under the curve (AUC) was calculated according to the trapezoid rule. Data were analyzed using Microsoft Excel 2007 (Microsoft, Redmond, WA, USA) and JMP 3.2 statistical software (SAS Institute, Cary, NC, USA). A p value of $<0.05$ was considered statistically significant.

\section{RESULTS}

A total of 10 volunteers (4 men, 6 women) participated in the study. Their age was $22.5 \pm 1.4$ years, and body mass index 24.4 \pm 4.1. Basic characteristics of BLS performance within both groups are summarized in table 1. Otherwise we did not detect any significant difference between groups.

Table 2 summarizes the results of energy expenditure calculations and rating of perceived exertion and sensation of general fatigue during both BLS protocols. Maximal $\mathrm{VO} 2 / \mathrm{kg}$ reached during BLS protocol was significantly higher when performing SBLS than CO-BLS. Expressed in metabolic equivalents, energy expenditure reached 
$5.8 \pm 0.6$ METs during CO-BLS and 6.6 \pm 1.1 METs during S-BLS ( $\mathrm{p}=0.049$ ). Calculation of AUCVO $2 / \mathrm{kg}$ min found significantly higher oxygen consumption associated with S-BLS than with CO-BLS. Overall, eight volunteers achieved higher maximal $\mathrm{VO} 2 / \mathrm{kg}$ and nine reached higher AUCVO $2 / \mathrm{kg}$ min when performing S-BLS than CO-BLS. Rating of perceived exer- tion with the Borg scale and sensation of general fatigue, revealed a higher degree of both parameters associated with CO-BLS than with S-BLS. Nine out of ten participants indicated S-BLS less exhaustive than CO-BLS. The only subjective reason consistently referred to was the possibility of a short rest during the pause for ventilations. Across both BLS protocols, the intensity of sensation of general fatigue positively correlated with the rate of perceived exertion $(\mathrm{r}=0.607, \mathrm{p}=0.005)$. Neither sensation of general fatigue nor perceived exertion correlated with $\mathrm{AUCVO} 2 / \mathrm{kg}$ min nor maximal reached $\mathrm{VO} 2 / \mathrm{kg}$.

Table 1. Characteristics of basic life support (BLS) performance in both groups.

\begin{tabular}{llll}
\hline VARIABLE & CO-BLS $(\mathbf{n}=\mathbf{1 0})$ & S- BLS $(\mathbf{n}=\mathbf{1 0})$ & $\mathbf{p}$ \\
\hline BLS duration $(\min \pm \mathrm{SD})$ & $21.0 \pm 8.7$ & $23.0 \pm 9.3$ & 0.434 \\
\hline reasons for BLS termination & & & 0.648 \\
\hline 30 min of quality BLS $(\mathrm{n})$ & 3 & 5 & 0.364 \\
\hline exhaustion $(\mathrm{n})$ & 5 & 2 & 1.000 \\
\hline insufficient quality of BLS $(\mathrm{n})$ & 2 & 3 & 0.112 \\
\hline compression rate $(\mathrm{n} / \mathrm{min} \pm \mathrm{SD})$ & $110.0 \pm 5.4$ & $114.0 \pm 5.8$ & 0.160 \\
\hline compression depth $(\mathrm{mm} \pm \mathrm{SD})$ & $52 \pm 4$ & $55 \pm 4$ & 0.970 \\
\hline total number of chest compressions $(\mathrm{n} \pm \mathrm{SD})$ & $2263 \pm 894$ & $2198 \pm 968$ & 0.997 \\
\hline$\%$ of adequate chest compressions $(\% \pm \mathrm{SD})$ & $83.5 \pm 12.8$ & $83.5 \pm 13.1$ & 0.646 \\
\hline compression-relaxation ratio $(\% \pm S D)$ & $48.1 \pm 2.9$ & $47.1 \pm 4.4$ & \\
\hline
\end{tabular}

BLS, basic life support; CO-BLS, compression only basic life support; S-BLS, standard basic life support.

Table 2. Energy expenditure calculations, rating of perceived exertion and sensation of general fatigue during CO-BLS and S-BLS procedure. * indicates statistically significant difference between the groups

\begin{tabular}{llll}
\hline VARIABLE & CO-BLS $(\mathbf{n}=\mathbf{1 0})$ & $\mathbf{S}-\mathbf{B L S}(\mathbf{n}=\mathbf{1 0})$ & $\mathbf{p}$ \\
\hline maximum $\mathrm{VO} 2 / \mathrm{kg}(\mathrm{ml} / \mathrm{kg} / \mathrm{min} \pm \mathrm{SD})$ & $20.17 \pm 2.14$ & $23.16 \pm 3.94$ & $0.049^{*}$ \\
\hline AUCVO $2 / \mathrm{kg} \min (\mathrm{ml} / \mathrm{min} 3 \pm \mathrm{SD})$ & $15.91 \pm 2.07$ & $18.90 \pm 3.13$ & $0.017^{*}$ \\
\hline maximum respiratory exchange ratio $($ mean $\pm \mathrm{SD})$ & $0.983 \pm 0.056$ & $0.971 \pm 0.064$ & 0.668 \\
\hline rating of perceived exertion (points $\pm \mathrm{SD})$ & $16.6 \pm 2.0$ & $13.8 \pm 1.2$ & $0.003^{*}$ \\
\hline rating of sensation of general fatigue (points $\pm \mathrm{SD})$ & $86.5 \pm 10.8$ & $75.0 \pm 14.3$ & 0.068 \\
\hline
\end{tabular}

AUC, area under the curve; AUCVO2/kg min, area under the curve of all VO2/kg measurements; CO-BLS, compression only basic life support; S-BLS, standard basic life support; $\mathrm{VO} 2 / \mathrm{kg}, \mathrm{ml} / \mathrm{kg} / \mathrm{min}$ relative oxygen consumption.

\section{DISCUSSION}

The main finding of this simulation study was the fact that S-BLS was associated with significantly higher energy expenditure but less sensation of general fatigue and lower perceived exertion intensity than the CO-BLS approach.

Although S-BLS is considered to be the gold standard in CPR, technically easier CO-BLS is allowed in certain situations. (1) Many laypeople are more willing to provide CO-BLS rather than S-BLS in the field. (5) However, the quality of BLS provided until the arrival of EMS is also a matter of physical capabilities of rescuers, especially when there is only one person on the scene. They are required to deliver chest compressions to a depth of $5-6 \mathrm{~cm}$ at a rate of $100-120 / \mathrm{min}$ with a compression:decompression ratio of 1:1. (6) In all aspects, it is a physically demanding activity and recently has become even more demanding than that recommended by ERC guidelines in 2005. (6)

Several manikin studies have revealed that BLS quality drops significantly during the first two minutes, especially the depth of compression, even when verbal feedback was provided in case of decreased compression depth. (7-13) This phenomenon is generally explained by the increasing rescuer's sensation of fatigue. Therefore, when there is more than one rescuer present, it is recommended to change the person delivering chest compressions every 2 min. (1) It has also been shown that there is a gradient of increasing sensation of fatigue depending on the increasing rate of compression-ventilation ratio in favor of compressions. (14) Sensation of fatigue is a subjective parameter. Nevertheless, very few studies evaluated objective measures 
reflecting physical limitation of BLS administration. Trowbridge et al. evaluated 20 healthy female volunteers performing both CO-BLS and S-BLS for 10 minutes and described a higher rating of perceived exertion for CO-BLS than for S-BLS, while differences in lactate values were not significant between the groups. (15) No difference in lactate values was found by Vaillancourt et al., when comparing S-BLS with the ratio of 30:2 and 15:2. (14) Shultz et al. compared energy expenditure measured by oxygen consumption of standard BLS and active compression-decompression cardiopulmonary resuscitation 5:1 performed for 10 minutes. They found it significantly higher in the active compression-decompression group $(\mathrm{p}<0.05)$. (16) Eventually, Jones et al. compared energy expenditure expressed as $\mathrm{O} 2$ consumption, compression effectiveness, and kinetics of the spine during simulated chest compression with the rescuer in different positions in 56 volunteers performing COBLS. Administering chest compressions while standing demanded more power but consumed less oxygen than doing so while kneeling, perhaps because fewer cardiac compressions delivered while standing were effective. (17)

These studies offer sophisticated insight into the process of BLS in terms of physical capabilities of providers. However, we still do not have the answers to the following questions: Do subjective parameters positively correlate with objective ones? Is there a difference between S-BLS and CO-BLS in energy expenditure, sensation of general fatigue and perceived exertion intensity? Can the anticipated decline in the quality of BLS be prevented, and if so, in both approaches? We believe that answering these questions will be beneficial for increasing the quality of clinical and simulation studies and hence for the entire optimization of BLS.

In our protocol, volunteers reached $6-7$ METs and a respiratory exchange ratio approaching 1,0 , which means that BLS, according to current guidelines, represents a moderate-to-vigorous level of physical activity on the border of the anaerobic threshold, comparable to snow shoveling, splitting wood, jogging or rowing. $(4,18,19)$ When compared with the results of Shultz et al., it indicates that mechanical optimizing BLS goes hand in hand with higher physical demands. (16)
We described that S-BLS was associated with significantly higher energy expenditure than CO-BLS. Our first hypothesis was not confirmed. The question remains what is the explanation for this phenomenon. It is obvious that chest compressions are the major component of BLS, determining energy demands during BLS. Purely in a mechanistic manner, we could suppose the more high-quality compressions per time unit, the higher the energy expenditure. However, in our study, participants delivered a comparable number of chest compressions in both groups. Another point of view is that CO-BLS has the features of continuous endurance, moderate to vigorous steady-state physical activity while S-BLS exhibits characteristics of high-intensity interval training (periods of intense chest compressions shortly interrupted by mouth-to-mouth ventilation). It has been shown that the latter pattern of physical activity increases energy expenditure during physical activity and postexercise oxygen consumption more than the former. (20-22) These findings fit our results.

Surprising for us was the detection of a mismatch between energy consumption and subjective feelings of fatigue and intensity of physical performance. Our second hypothesis was not confirmed either. The observation was supported by the finding that all but one volunteer expressly described S-BLS as less exhausting than CO-BLS. Probably, it can be explained by the general statement of volunteers that a short pause for ventilation was perceived as a time for rest. This means that regular expectations of intervals for a short rest can have a significant positive psychological effect on the perception of expended physical activity, although it is associated with higher energy expenditure. The question is what to prefer in the clinical setting of telephone-guided CPR when a long time until ambulance car arrival is anticipated, rather S-BLS, because it is less energy consuming than CO-BLS, or vice versa CO-BLS, because it is associated with less sensation of fatigue. Because the level of physical activity during BLS is not maximal but moderate-to-vigorous, we suppose that in young rescuers S-BLS should always be considered. It is known, that bystanders of cardiac arrests are usually people of similar age as the victims. (23) The emergency medical dispatchers may probably take into consideration age and physical capabilities of rescuers when providing telephone instructions for CPR. It is not clear if rescuers with lower physical fitness (e.g. the elderly, bystanders with coronary artery disease, heart failure or other illnesses) should be instructed differently, and if exercise during CPR itself is still an acceptable risk for them. Unfortunately, these questions could not been answered on the basis of our results. We were also unable to recognize if the proven mismatch between energy expenditure and subjective feelings of fatigue was linked to other demographic groups, other than young students, and whether there are any differences between men and women. However, we consider it important that this issue has been raised. It opens additional possibilities for optimizing BLS and telephone-guided CPR in the sense of tailoring the procedure to specific rescuers.

Our findings also indicate that the quality of BLS may be favorably influenced by positive, active motivation provided at regular intervals and led by a non-resuscitating observer, even in cases where BLS is carried out precisely. Using this approach, we reached a consistently higher quality of CPR for longer periods than in other studies with volunteers. $(9,24)$ We believe that the principle of automatic, periodic, positive motivation by an EMS dispatcher can be easily transferred to telephone-guided CPR protocols.

\section{STUDY LIMITATIONS}

Our clinical study has several limitations. Some of them have already been mentioned. First, the study was performed on a small sample of volunteers. Second, all volunteers were healthy young people and their results may not be reproducible to other age groups. Third, simulation of mouth-to-mouth ventilations by breathing into a facemask may have affected the measured parameters, but we believe that this bias is clinically irrelevant. Fourth, simulation is different compared to real CPR in the field. Facing a real OHCA, especially one affecting relatives, causes a higher degree of motivation in order to save someone's life. Release of stress hormones in such settings can expectedly suppress fatigue, and modify energy expenditure to an unknown extent. 


\section{CONCLUSIONS}

We conclude that the results of our study do not change the current approach to BLS in general. Nevertheless, evaluation of energy expenditure, sensation of general fatigue and perceived exertion intensity dur- ing standard BLS and compression only BLS in our pilot simulation study revealed that while S-BLS was associated with significantly higher energy expenditure than CO-BLS, study participants reported a lower degree of sensation of general fatigue and perceived exertion intensity during $\mathrm{S}$ -
BLS compared to CO-BLS. In our study, repeated positive verbal motivation of volunteers led to long-term high-quality CPR. Further studies are warranted to describe the process of BLS from the more detailed perspective of its providers.

\section{REFERENCES}

1. Koster RW, Baubin MA, Bossaert LL, Caballero A, Cassan P, Castrén M, et al. European Resuscitation Council Guidelines for Resuscitation 2010 Section 2. Adult basic life support and use of automated external defibrillators. Resuscitation 2010;81:1277-92.

2. Pinheiro Volp AC, Esteves de Oliveira FC, Duarte Moreira Alves R, Esteves EA, Bressan J. Energy expenditure: components and evaluation methods. Nutr Hosp 2011;26:430-40.

3. Borg G. Studia Psychologica et Paedagogica, Series altera, Investigationes XI. Lund, Sweden: Gleerup; 1962.

4. Ainsworth BE, Haskell WL, Herrmann SD, Meckes N, Bassett DR, Tudor-Locke C, et al. 2011 Compendium of Physical Activities: a second update of codes and MET values. Med Sci Sports Exerc 2011;43:1575-81.

5. Cho GC, Sohn YD, Kang KH, Lee WW, Lim KS, Kim W, et al. The effect of basic life support education on laypersons' willingness in performing bystander hands only cardiopulmonary resuscitation. Resuscitation 2010;81:691-4.

6. Handley AJ, Koster R, Monsieurs K, Perkins GD, Davies S, Bossaert L. European Resuscitation Council guidelines for resuscitation 2005. Section 2. Adult basic life support and use of automated external defibrillators. Resuscitation 2005;67 Suppl 1:S7-23.

7. Heidenreich JW, Berg R a, Higdon T a, Ewy G a, Kern KB, Sanders AB. Rescuer fatigue: standard versus continuous chest-compression cardiopulmonary resuscitation. Acad Emerg Med 2006;13:1020-6.

8. Neset A, Birkenes TS, Myklebust H, Mykletun RJ, Odegaard S, Kramer-Johansen J. A randomized trial of the capability of elderly lay persons to perform chest compression only CPR versus standard 30:2 CPR. Resuscitation 2010;81:887-92.

9. McDonald CH, Heggie J, Jones CM, Thorne CJ, Hulme J. Rescuer fatigue under the 2010 ERC guidelines, and its effect on cardiopulmonary resuscitation (CPR) performance. Emerg Med J 2013;30:623-7.

10. Gutwirth H, Williams B, Boyle M. Rescuer fatigue in cardiopulmonary resuscitation: a review of the literature. JEPHC 2009;7:19-27.

11. Odegaard S, Saether E, Steen PA, Wik L. Quality of lay person CPR performance with compression: ventilation ratios 15:2, 30:2 or continuous chest compressions without ventilations on manikins. Resuscitation 2006;71:335-40.

12. Jones CM, Owen A, Thorne CJ, Hulme J. Comparison of the quality of basic life support provided by rescuers trained using the 2005 or 2010 ERC guidelines. Scand J Trauma Resusc Emerg Med 2012;20:53.

13. Deschilder K, De Vos R, Stockman W. The effect on quality of chest compressions and exhaustion of a compression-ventilation ratio of 30:2 versus 15:2 during cardiopulmonary resuscitation-a randomised trial. Resuscitation 2007;74:113-8.

14. Vaillancourt C, Midzic I, Taljaard M, Chisamore B. Performer fatigue and CPR quality comparing 30:2 to 15:2 compression to ventilation ratios in older bystanders: A randomized crossover trial. Resuscitation 2011;82:51-6.

15. Trowbridge C, Parekh JN, Ricard MD, Potts J, Patrickson WC, Cason CL. A randomized cross-over study of the quality of cardiopulmonary resuscitation among females performing 30:2 and hands-only cardiopulmonary resuscitation. BMC Nurs 2009;8:6-18.

16. Shultz JJ, Mianulli MJ, Gisch TM, Coffeen PR, Haidet GC, Lurie KG. Comparison of exertion required to perform standard and active compression-decompression cardiopulmonary resuscitation. Resuscitation 1995;29:23-31.

17. Jones AYM, Lee RYW. Rescuer's position and energy consumption, spinal kinetics, and effectiveness of simulated cardiac compression. Am J Crit Care 2008;17:417-25.

18. Ainsworth BE, Haskell WL, Whitt MC, Irwin ML, Swartz a M, Strath SJ, et al. Compendium of physical activities: an update of activity codes and MET intensities. Med Sci Sports Exerc 2000;32:S498-504.

19. Solberg G, Robstad B, Skjonsberg OH, Borchsenius F. Respiratory gas exchange indices for estimating the anaerobic threshold. J Sports Sci Med 2005;4:29-36.

20. Burgomaster K a, Howarth KR, Phillips SM, Rakobowchuk M, Macdonald MJ, McGee SL, et al. Similar metabolic adaptations during exercise after low volume sprint interval and traditional endurance training in humans. J Physiol 2008;586:151-60.

21. Larsen I, Welde B, Martins C, Tjønna AE. High- and moderate-intensity aerobic exercise and excess post-exercise oxygen consumption in men with metabolic syndrome. Scand J Med Sci Sports 2014;24:e174-9.

22.Zuniga JM, Berg K, Noble J, Harder J, Chaffin ME, Hanumanthu VS. Physiological responses during interval training with different intensities and duration of exercise. J Strength Cond Res 2011;25:1279-84.

23. Birkenes TS, Myklebust H, Neset A, Kramer-Johansen J. Quality of CPR performed by trained bystanders with optimized pre-arrival instructions. Resuscitation 2014;85:124-30.

24. Heidenreich JW, Bonner A, Sanders AB. Rescuer fatigue in the elderly: standard vs. hands-only CPR. J Emerg Med 2012;42:88-92.

25. Acknowledgments

26. Supported by the programme PRVOUK (Research Area Development Programmes at Charles University) P37/02 and by Ministry of Health, Czech Republic - conceptual development of research organization (UHHK, 00179906). 